\title{
BRACING TYPE PURE ALUMINIUM STIFFENED SHEAR PANELS: AN EXPERIMENTAL STUDY
}

\author{
Gianfranco De Matteis ${ }^{1, *}$, Giuseppe Brando ${ }^{1}$, Simeone Panico ${ }^{2}$ and Federico M. Mazzolani ${ }^{2}$ \\ ${ }^{I}$ Department of Design Rehabilitation and Control of Architectural Structures (PRICOS), \\ University of Chieti-Pescara "G d'Annunzio", Italy \\ ${ }^{2}$ Department of Engineering Structures (DIST), University of Naples "Federico II", Italy \\ *(Corresponding author: E-mail: demattei@unina.it)
}

\begin{abstract}
Shear panels represent nowadays one of the most suitable devices for protecting steel medium-high rise buildings subjected to both seismic and aeolian actions. In particular, the employment of stiffened plates made of low yield strength material provides an effective dissipative capability to the whole structure, which can be controlled as a design parameter by choosing appropriate panel dimensions and varying the stiffeners arrangement. The main purpose of this paper is to illustrate the principal results of an experimental campaign carried out on two bracing type pure aluminium shear panels (BTPASPs) tested under a diagonal cyclic force. Selected specimens are stiffened by means of welded aluminium ribs and are characterized by different aspect ratio values. A comparison between the obtained structural responses, in terms of experimental evidences, energy dissipation capability, hardening ratio, secant global stiffness and equivalent viscous damping ratio, is provided.
\end{abstract}

Keywords: Pure aluminium, shear panels, dissipative devices, passive seismic control, cyclic response, FEM model

\section{INTRODUCTORY REMARKS}

The use of passive energy dissipation (PED) metallic devices, placed in the primary framed structures of both steel and r.c. medium-high buildings for their protection during a seismic event, has found a lot of application in the last few decades. Among these, shear panels represent one of the most suitable tool to improve the structural performance in terms of both energy dissipation capability and lateral stiffness (Dargush and Soong [1]). In fact, the possibility of exploiting their effective post-buckling behaviour, which develops by means of a tension field shear mechanism, allows to get benefit from the ductile feature of the base material. In addition, the dissipative capability of these devices can be improved by using a low yield strength base material and applying adequate flexural stiffeners to the basic plate in order to have a very good hysteretic behaviour, with the energy dissipation function activated already for very small drift angles and pinching effects arising only when high plastic shear strain demands occur. Furthermore, it has to be considered that due to moderate initial shear stiffnesses, these systems could also improve the global structural performances with reference to the serviceability limit state requirements.

Many researches have been performed in order to find suitable analytical and physical hysteretic models able to simulate the dissipative features of stiffened shear plates (Chen et al. [2]). However, the main efforts have been devoted to solve two crucial aspects. The former concerns the technological choice of a base material characterized by a low yielding point, in such a way, consistently with the "capacity design" criteria, hysteretic properties of dampers could be activated when the primary structure is still in the elastic field. The latter is related to the pursuit of design charts finalized to optimise the geometry of the device in terms of type and location of the stiffeners. From both these points of view, the studies carried out in Japan in the earlier nineties should be mentioned (Nakashima [3-5]). In particular, the choice of a steel with a low carbon content, characterized by both a low yield stress point and a high ductility value, has been proposed, leading to the use of the well known LYSW (Low Yield Strength Wall) devices. 
On the other hand, this type of material is not produced and commercialised in the European market and research of alternative solutions has been carried out. In particular, an innovative type of full-bay shear panels (see Figure 1-a and Figure 1-b) made of pure aluminium alloy as base material has been recently proposed at the University of Naples "Federico II", where several studies, from both the experimental (De Matteis et al. [6]) and the numerical (De Matteis et al. [7 8]) points of view, have been implemented. Such a material is characterized by a nominal yield strength significantly lower than the LYS steel, with a similar elongation at rupture, provided that preliminarily heat treatment processes are applied. The obtained results have shown a very attractive performance, with a high dissipative capability, which is testified by the large hysteretic cycles obtained. From the other hand, some slipping phenomena for low lateral displacement values have been observed limiting the performance of the systems.
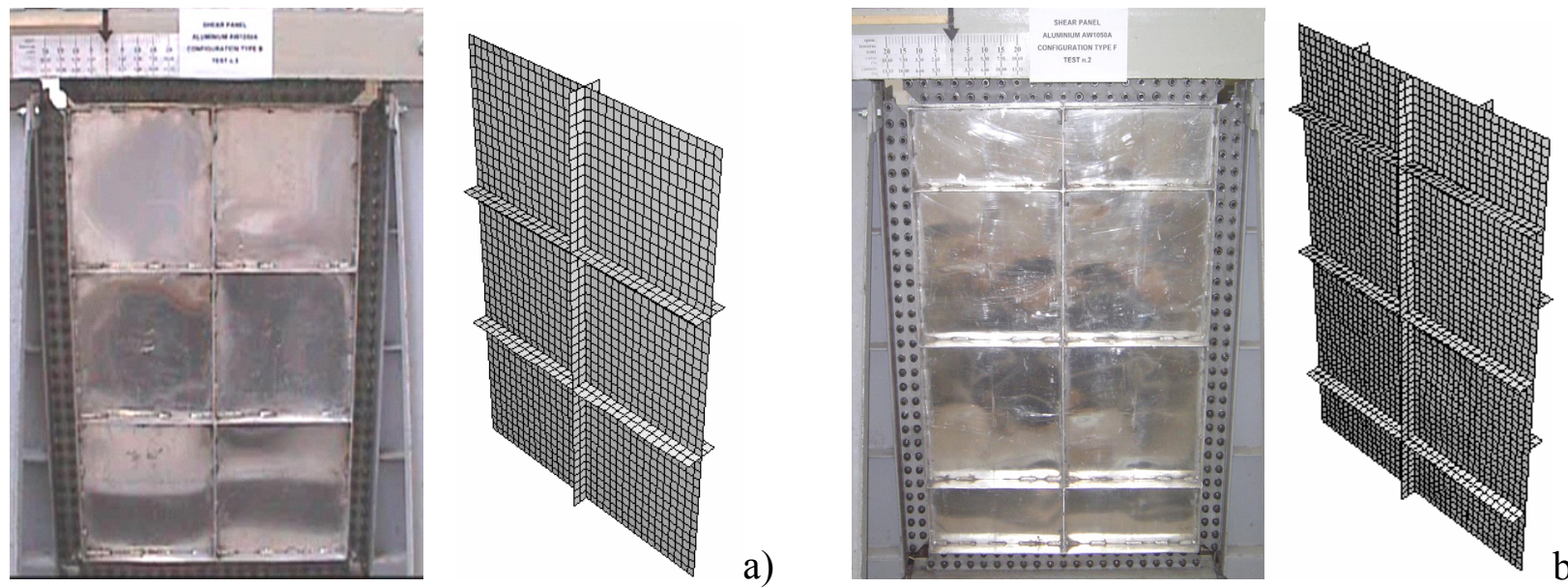

b)

Figure 1. Experimental and Numerical Models of Tested Full-bay Pure Aluminium Shear Panels

In order to increase the local deformation demand of shear panels for reduced inter-story drifts of the primary framed structure, exploiting the high ductility of the base material, the bracing type configuration of shear panel has been adopted (see Figure 2). It is characterised by reduced dimensions of shear panels respect to the surrounding frame field, so to increase the ratio between the shear deformation of the panel and the developed interstory drift.
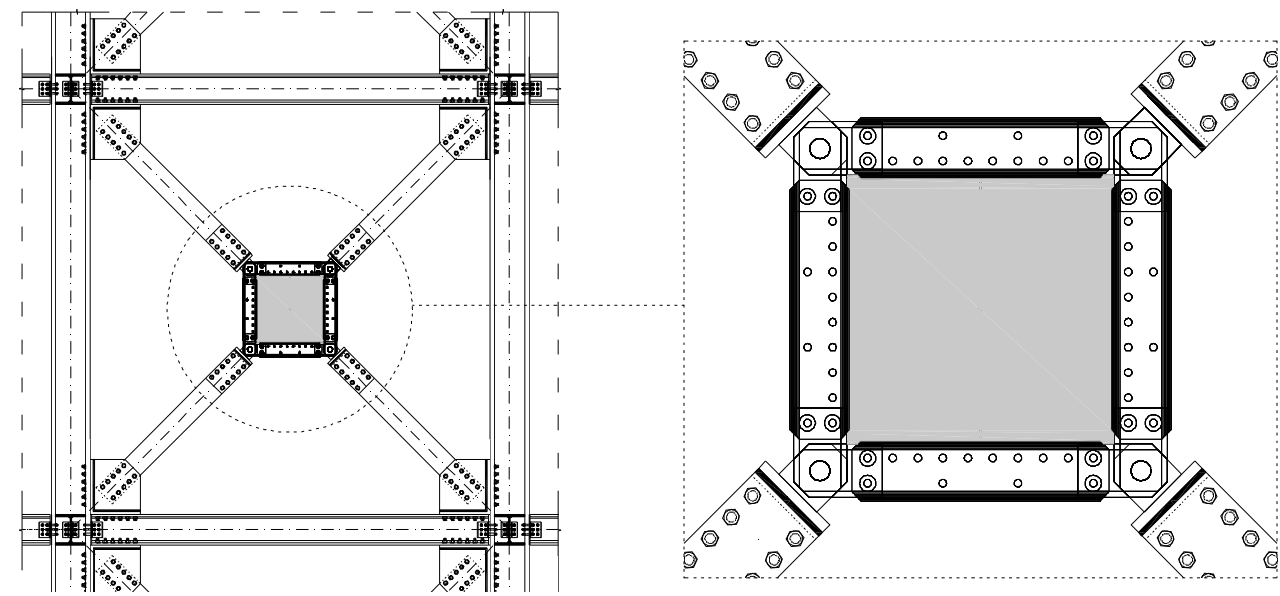

Figure 2. Bracing Type Shear Panel Configuration 
Bracing type pure aluminium shear panels (BTPASPs) may therefore be effectively used as passive protection device in both steel and r.c. moment resisting frames. In order to evaluate the influence of the main geometrical parameters on the cyclic behaviour, two shear panel specimens characterises by different aspect ratio values have been experimentally tested by applying a diagonal cyclic loading procedure. The main obtained results and the experimental evidences of the tests are shown in the current paper.

\section{THE PROPOSED SYSTEMS}

\subsection{Basis}

The innovative aspects of the proposed material, which is an alloy characterized by a high percentage of pure aluminium never employed before in the structural engineering field, lead to a deep investigation of its mechanical features with the aim of attaining a better knowledge of its behaviour for both monotonic and cyclic loading.

On the other hand, as far as the design process of the shear panels is concerned, the choice of stiffener configuration represents a key aspect to determine the device performance. In fact, a shear plate where ribs are correctly arranged provides a better hysteretic behaviour, the response of the system being mainly characterised, at least for a huge part of the plastic field, by a pure shear resistant mechanism. Moreover the flexural stiffness of the stiffeners must be set in appropriate way, so that global buckling phenomena, which negatively influence the system response, do not occur. On the basis of the above considerations, a study based on a twofold approach has been developed: (1) inspection of the mechanical behaviour of the base material, (2) investigation on the most appropriate stiffener configuration to be adopted in relation to the expected structural demand.

\subsection{The Adopted Material}

The tested bracing type pure aluminium shear panels have been fabricated with plates and stiffeners made of aluminium alloy AW1050 A H24, having a thickness of $5 \mathrm{~mm}$. The chemical composition and nominal mechanical properties of the adopted material are listed in Table 1.

Table 1. Chemical Composition and Mechanical Properties of the Adopted Aluminium Alloy 1050A H24

\begin{tabular}{|c|c|c|c|}
\hline \multicolumn{2}{|c|}{$\begin{array}{l}\text { Commercial } \\
\text { denomination }\end{array}$} & & Impurities \\
\hline \multirow[t]{2}{*}{ Aluminium } & $99.50 \%$ & & $\begin{array}{c}0.02 \% \mathrm{Cu}, 0.40 \% \mathrm{Fe} \\
0.31 \% \mathrm{Si}, 0.07 \% \mathrm{Zn}, \\
0.02 \% \mathrm{Tl}, 0.02 \% \text { other }\end{array}$ \\
\hline & & $\begin{array}{l}\text { Mechanical } \\
\text { properties }\end{array}$ & \\
\hline \multicolumn{2}{|c|}{$\begin{array}{c}\text { Tensile Strength } \\
{[\mathrm{Mpa}]}\end{array}$} & $\begin{array}{c}\text { Yield Strength } \\
{[0.2 \% \text { offset, MPA }]}\end{array}$ & $\begin{array}{c}\text { Elongation on } 5 \mathrm{~cm} \\
{[\%]}\end{array}$ \\
\hline \multicolumn{2}{|c|}{$70-100$} & $30-70$ & $20-40$ \\
\hline
\end{tabular}


Preliminary uniaxial tensile tests have been performed according to the specifications provided by RILEM [9]. The obtained results (De Matteis et al. [6]) have shown a higher conventional yielding strength $\left(f_{02}\right)$ and a lower ductility than the nominal ones. To enhance the desirable ductile property and reduce the yield stress, a heat treatment process has been therefore carried out. The specimens have been submitted to a cycle of heat treatment characterized by different phases with constant temperature, each one having a duration of four hours. Successive tensile tests have shown a considerable improvement of ductile properties with a significant reduction of yield strength and an increase of ultimate elongation. In particular a conventional yield stress $f_{02}$ of $18 \mathrm{MPa}$ and a ductility of around $50 \%$ have been registered. For the sake of clarity, the comparison, in terms of strain-stress relationship, between the adopted pure aluminium alloy after the applied heat treatment, a common aluminium alloy (AW5154 A) and a typical low strength steel is shown in Figure 3.

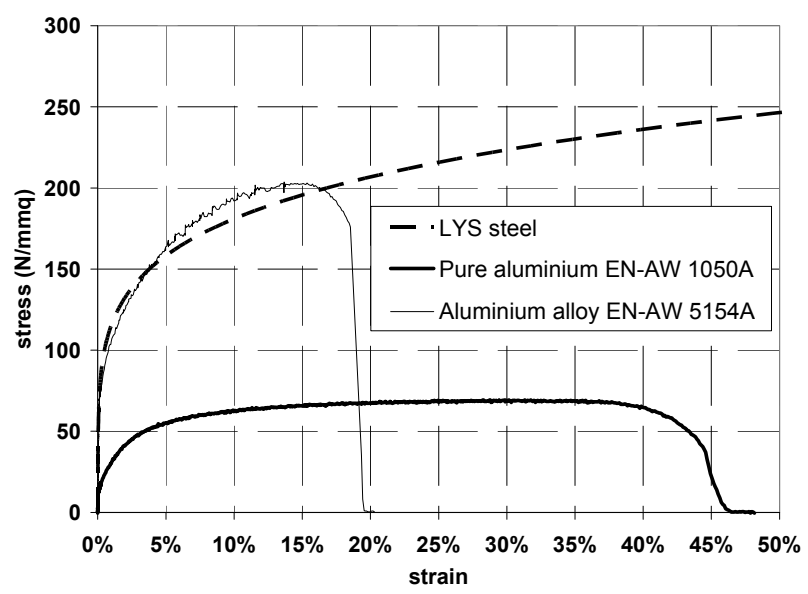

Figure 3. Comparison of Stress-strain Relationship Between Considered Low-strength Materials

In addition, as already emphasised in previous studies (Nakashima [3], Katayama [10]), the characterization of the cyclic behaviour of a low yield strength material is very important, as the isotropic hardening component could influence favourably the dissipative effect of the devices made of this type of material, since a reinflating of the hysteretic cycles is usually detectable. For this reason, specific tensile-compression cyclic tests have been carried out on pure aluminium specimens equipped with a steel "jacket" able to inhibit out-of-plane deformations due to buckling phenomena in compression (see Figure 4). The obtained results have shown an important dissipative behaviour (see Figure 5-a) characterized by full hysteretic cycles, a substantial iso-resistance for each displacement level and the existence of an isotropic hardening component (see Figure 5-b). However, as far as the cyclic degradation is concerned, performed tests did not allow to yield final conclusions, since testing specimens showed unexpected local buckling phenomena, which invalidated the experimental results for displacements higher than $+15 \mathrm{~mm}$ (see Figure 5-c, 5-d, 5-e). 

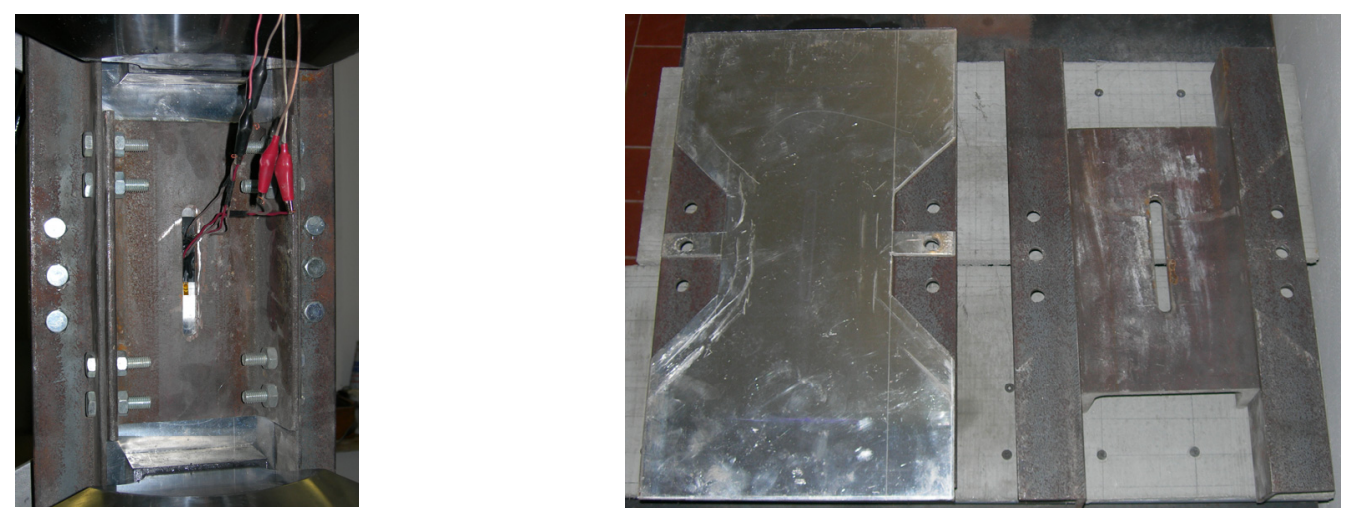

Figure 4. Buckling Inhibited Pure Aluminium Specimen
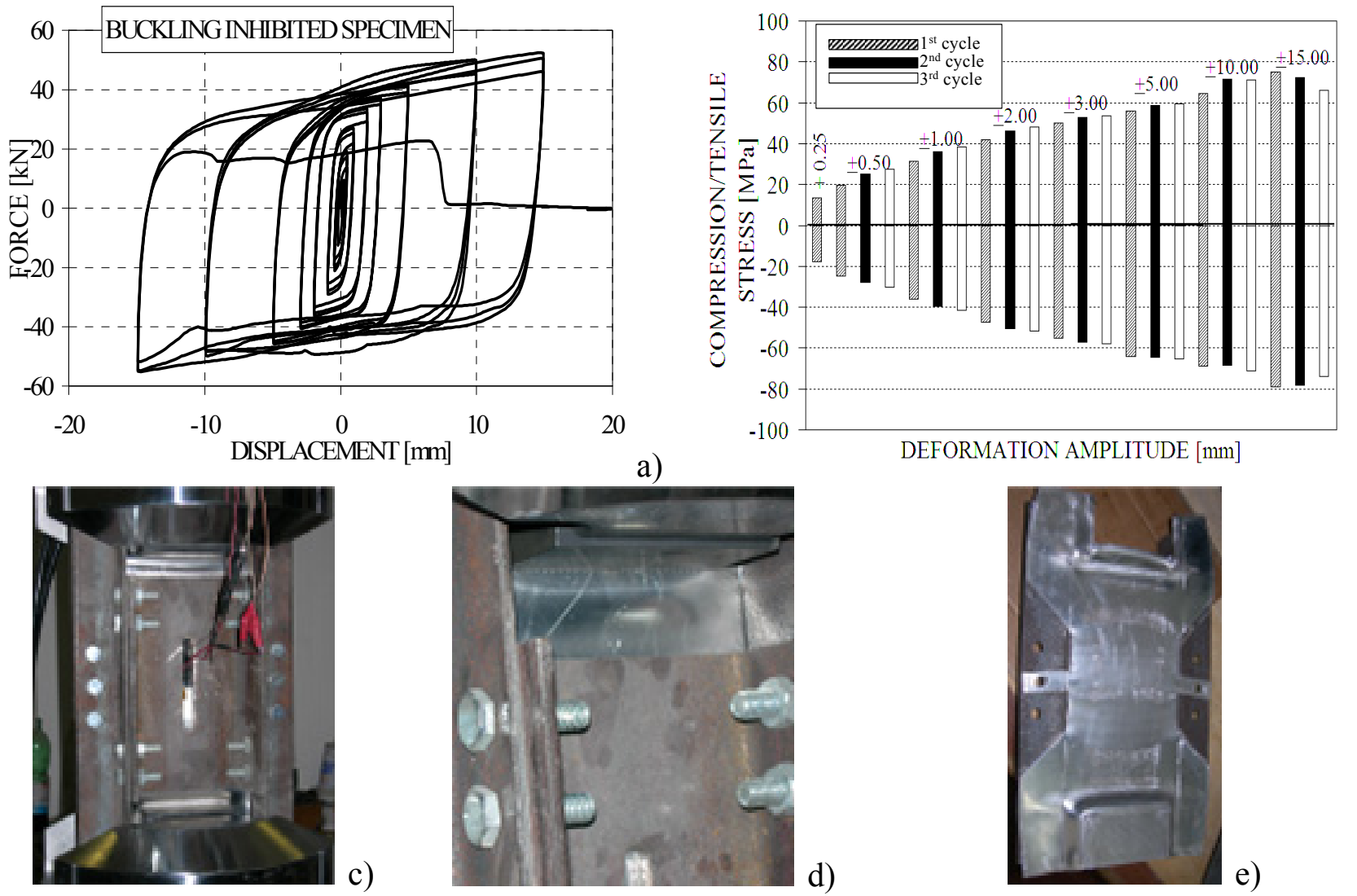

b)

Figure 5. Buckling Inhibited Pure Aluminium Specimen: (a) Hysteretic Behaviour;

(b) Strength for Each Cycle; (c-d-e) Collapse Mode

\subsection{The Tested Specimens}

The two tested shear panels, which henceforth will be indicate as "type 1" and "type 2", are both characterized by global dimensions of 500 by $500 \mathrm{~mm}$ and a plate thickness of $5 \mathrm{~mm}$. They are equipped with welded rectangular-shaped ribs, equally placed on the two faces of the panels, whose depth is $60 \mathrm{~mm}$ and which are made of the same material and thickness of the basic plates. The two specimens have been inserted into a pin jointed steel framework and are linked to its channel elements along their edge by means of tightened steel bolts. The stiffeners arrangement has been chosen in such a way to have a slenderness ratio $a_{w} / t_{w}$ equal to 100 and 50 for shear panel "type 1" 
and "type 2", respectively (see Figure 6). The spacing and depth of the ribs have been selected to fulfil the requirements provided by the European Standard to ensure shear buckling of the plate after shear yielding (Höglund et al. [11]). In fact, Eurocode 9 [12] provides the following limit value (Eq. 1) of a slenderness parameter $l_{\mathrm{w}}$ under which shear buckling resistance is higher than the yielding resistance:

$\bar{\lambda}_{w}=\frac{0.83}{\eta}=0.69$

where the $\eta$ factor, for which Eurocode 9 fixes a superior limit value of 1.2 , is given by the following expression (Eq. 2):

$\eta=0.7+0.35 \cdot \frac{f_{a w}}{f_{o w}}$
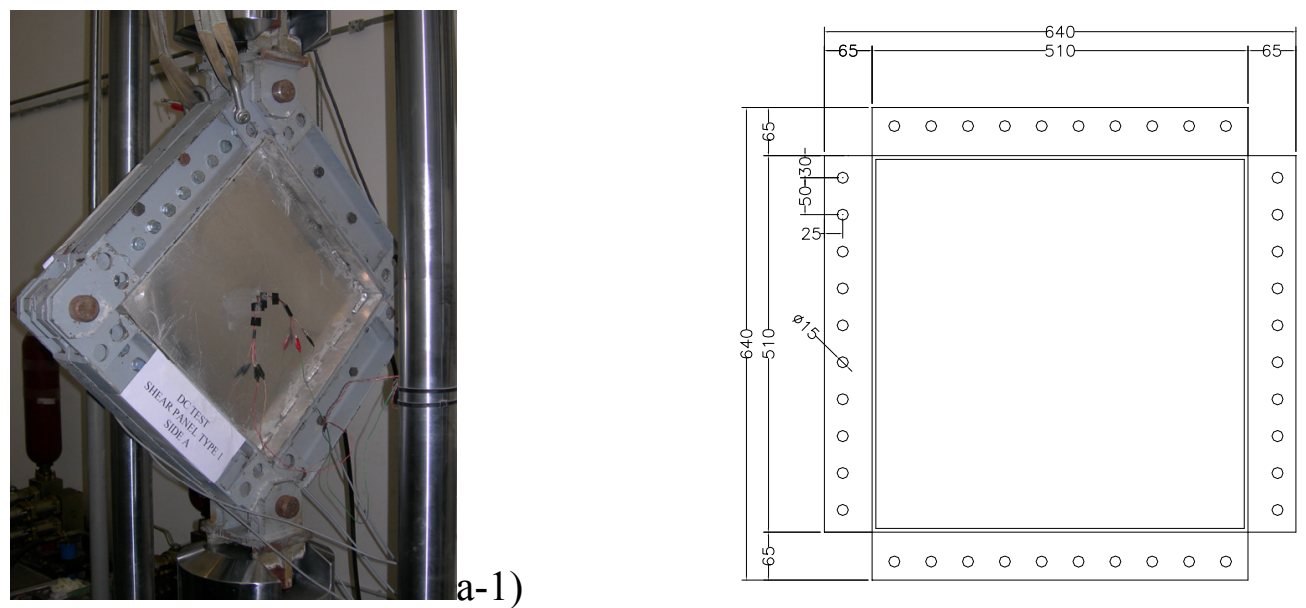

a-2)
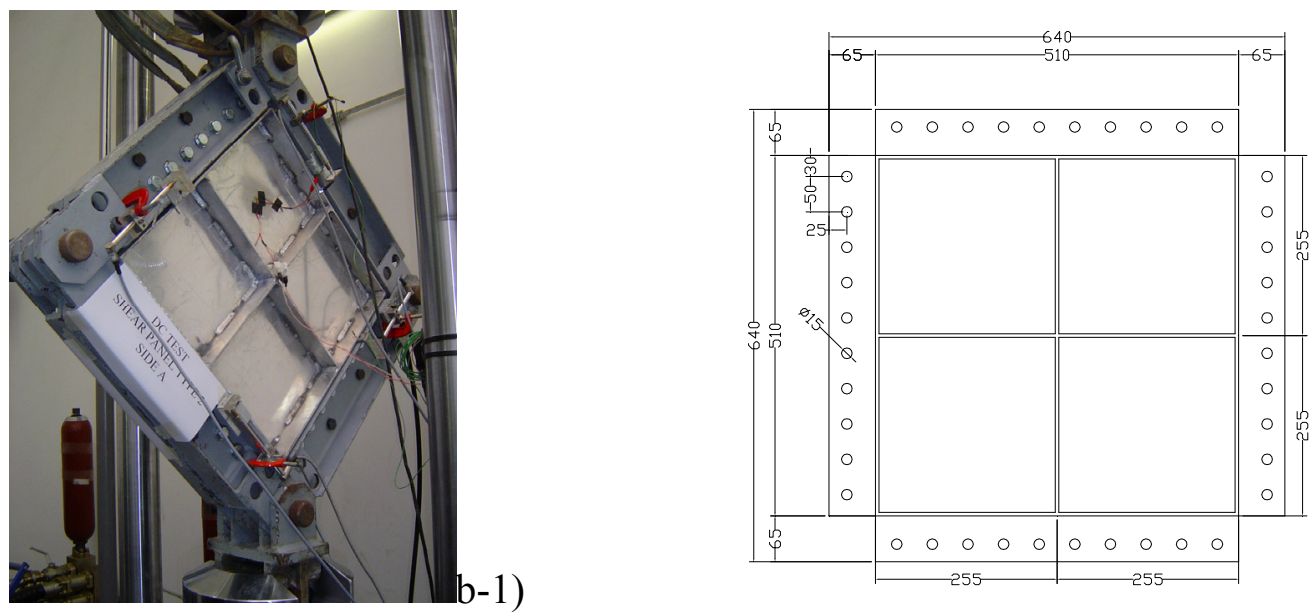

b-2)

Figure 6. Testing Specimens: (a) Shear Panel "type 1"; (b) Shear Panel "type 2"

In the case being, the $\eta$ factor has been conventionally assumed equal to 1.2 , even though the hardening ratio a $\left(f_{\text {aw }} / f_{\text {ow }}\right)=3$ would lead to a corresponding $h$ value equal to 1.75.

Hence, it is possible to observe that studied shear panels in such design assumptions guarantee a slenderness parameter value lower than the limit one. In particular, the slenderness parameters for 
the proposed panels, considering that panel "type 1" could be related to a "plate girders with web stiffeners at support" while panel "type 2" to a "plate girders with intermediate web stiffeners", are given by Eq. 3 and Eq. 4, respectively:

- Shear panel "type1":

$$
\begin{aligned}
& \lambda_{w, 1}=0.35 * \frac{b_{w}}{t_{w}} * \sqrt{\frac{f_{0}}{E}}=0.66 \\
& \lambda_{w, 2}=\frac{0.81}{\sqrt{k_{\tau}}} * \frac{b_{w}}{t_{w}} * \sqrt{\frac{f_{0}}{E}}=0.55
\end{aligned}
$$

As far as the effectiveness of the flexural stiffness of panel type 2 longitudinal and intermediate ribs is concerned, it is possible to observe that the adopted depth of $60 \mathrm{~mm}$ fulfils the Eurocode 9 requirements in order to classify them as rigid supports. In particular, since the panel has an aspect ratio $a / h_{w}$ equal to 0.5 (smaller than the limit value of $\sqrt{2}$ ), the minimum second moment of area of the ribs to be not involved in global buckling phenomena of the shear plate is:

$$
I_{s t, \mathrm{lim}, 2}=1.5 \cdot \frac{{h_{w}{ }^{3} \cdot t_{w}{ }^{3}}_{a^{2}}}{a^{2}}=375000 \mathrm{~mm}^{4}
$$

It is worthy noting that in the case being the second moment of area is equal to $720000 \mathrm{~mm}^{4}$, therefore about twice the above limit value.

On the other hand, similar considerations are not necessary for the panel "type 1", since the rigid end posts are realized on the four edges by means of the rigid perimeter beams of the frame in which the plate is installed.

\section{THE EXPERIMENTAL TESTS}

\subsection{Loading Protocol and Applied Measurement Devices}

The loading protocol used for tests has been set on the basis of the ECCS-CECM [13], which, for cyclic procedures, retrieves a load history essentially based on some cycles in the elastic range and then on three cycle repetitions for progressively increasing displacement amplitudes, defined as integer multiples of $v_{y}$, which is the displacement corresponding to the attainment of yielding. In the case being, the conventional yielding tangential stress can be fixed on the based of the conventional yielding tangential stress stated as in (Eq. 6)

$\tau_{y}=\frac{f_{0.2}}{\sqrt{3}}=10.39 \mathrm{MPa}$

Such a stress value corresponds to a shear strain equal to $g=0.04 \%$, hence the diagonal displacement $v_{y}$ corresponding to the attainment of yielding can be evaluate as about $0.5 \mathrm{~mm}$. Since this value is very small, the first steps of the above procedure have been eliminated, while the other ones have been left unvaried, so that the adopted testing protocol for each panel type is the one shown in Figure 7-a, where the ordinate indicates the applied diagonal displacement on the top of the surrounding frame (see Figure 7-b). 


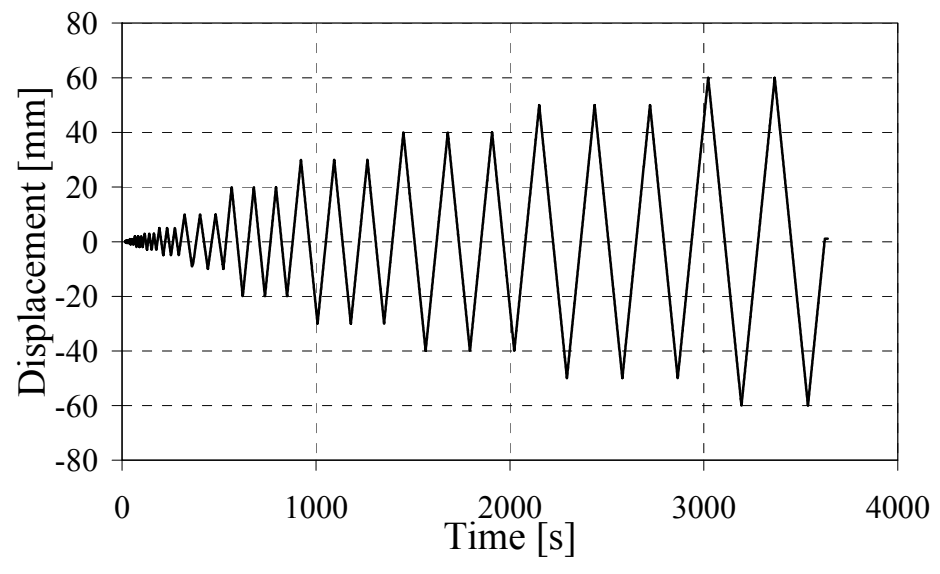

a)

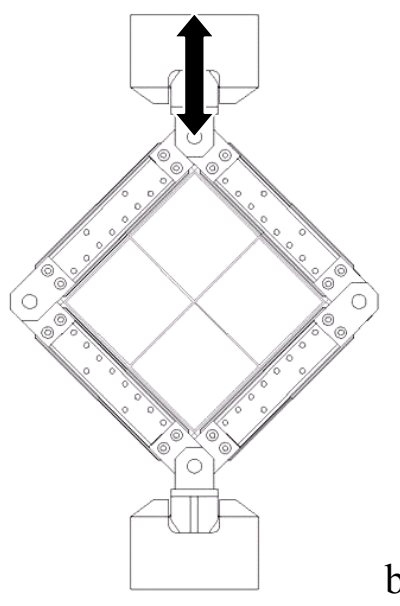

Figure 7. Loading Scheme (a) and Applied Displacement History (b)

Such a displacement was measured by means of a mechanical transducer whose signal was fed back in real time to the controller as an instrument of supervision of the jack motion. Also, four mechanical transducer have been placed on the perimeter of the panels, to measure the relative motion between the panel edges and the frame elements and therefore to understand which is the influence of the frame-to-plate bolted connections on the behaviour of the whole system. This is a very important aspect, as previous experimental campaigns carried out on similar devices shown that bolted connections may represent the weak point of the system. To monitor the strain behaviour of tested shear panels, a pair of uniaxial strain gauges were also glued at the centre of the panel for panel "type 1" and at the centre of a sub panel of panel "type 2". The applied measurement instrumentation and its arrangement and location can be observed in Figure 6.

\section{TEST RESULTS, EXPERIMENTAL EVIDENCES AND DISCUSSION}

In this section, a careful presentation and discussion of both the obtained test results and experimental evidence is provide. In Figure 8, the response of the two shear panel types is shown in terms of obtained force-displacement hysteretic cycles, while the relevant collapse modes are given in Figure 9.
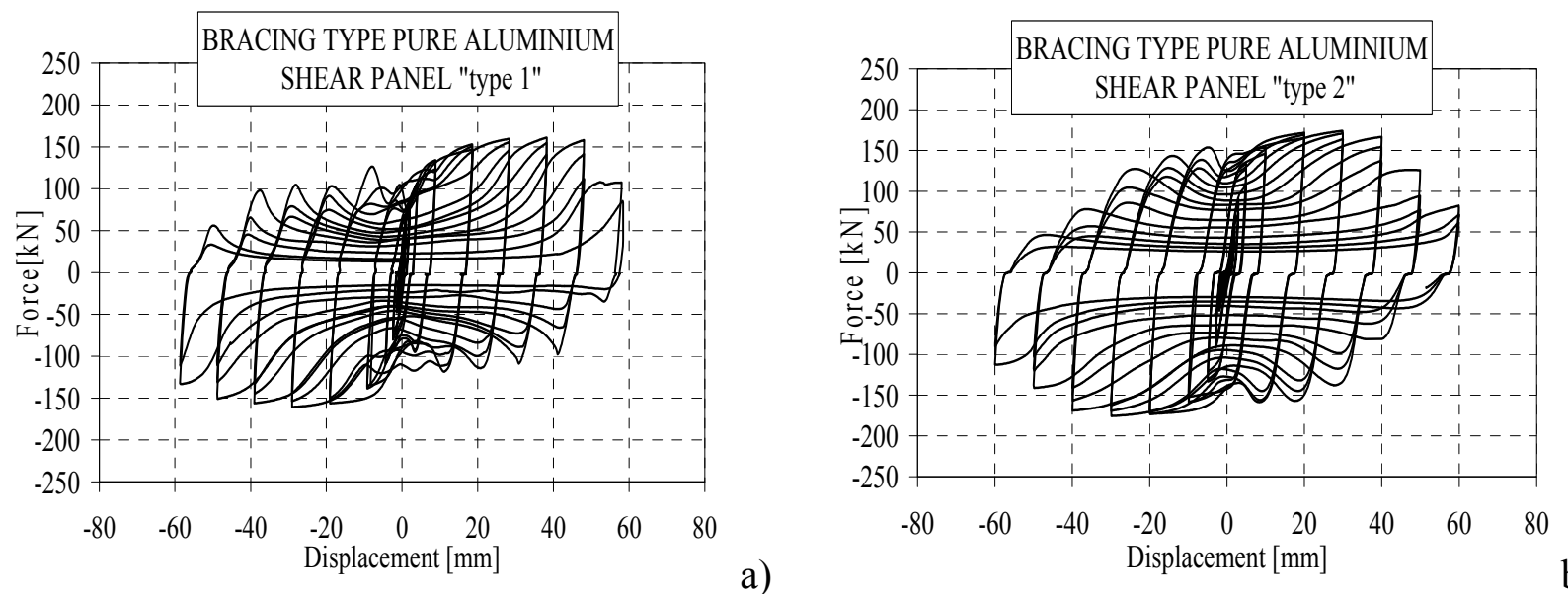

a)

b)

Figure 8. Hysteretic Cycles: (a) Shear Panel "type 1"; (b) Shear Panel "type 2" 

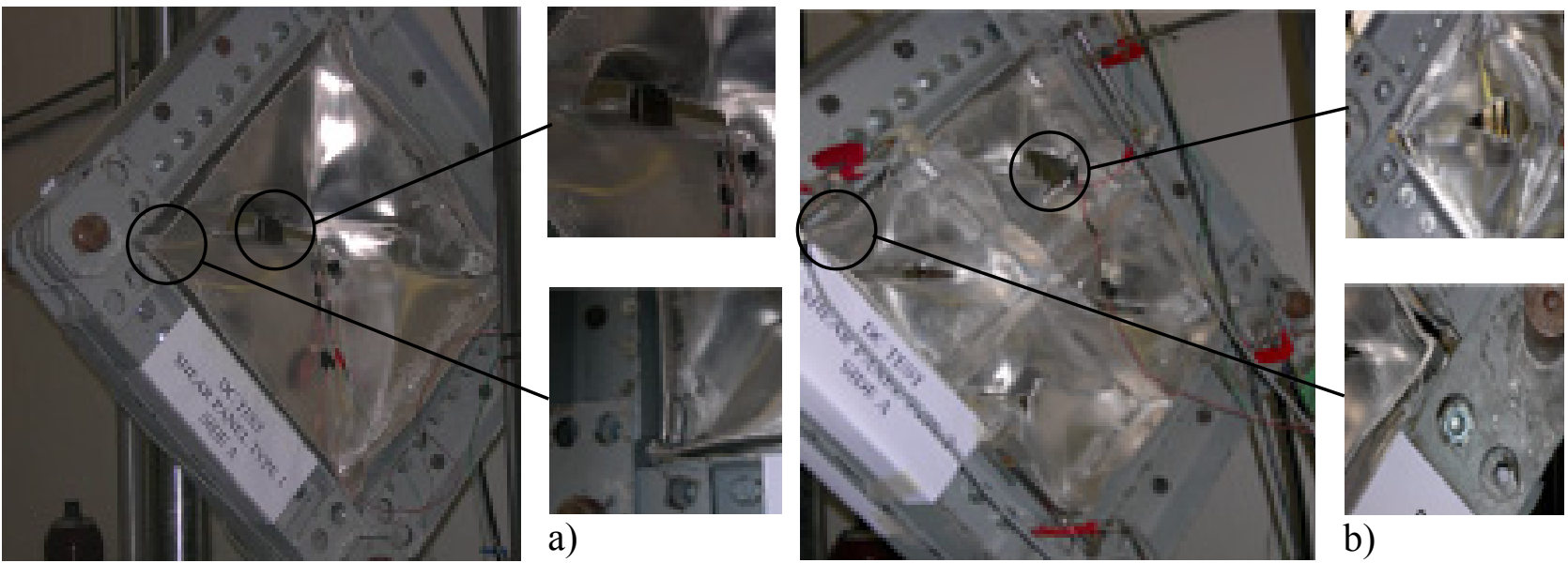

b)

Figure 9. Collapse Modes: (a) Shear Panel "type 1"; (b) Shear Panel "type 2"

It should be noted that the two shear panels exhibited unexpected, but not so important slipping phenomena for very small force levels, which were probably due to the initial arrangement of the testing system. Anyway, it is evident that shear panel "type 2" provides a better hysteretic behaviour, with fatter cycles and larger resistant capacity. In fact, more important pinching effects characterize the obtained hysteretic cycles for shear panel "type 1". These aspects are clearly emphasised considering the comparisons of the two panel types in terms of energy dissipation, hardening ratio, secant global stiffness and equivalent viscous damping ratio, as shown in Figure 10, where these parameters are expressed as function of the applied shear strain value.

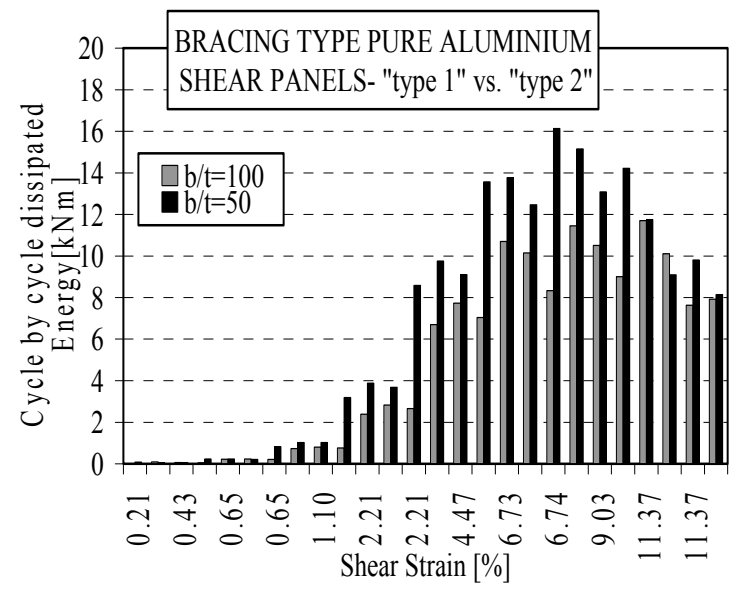

a)
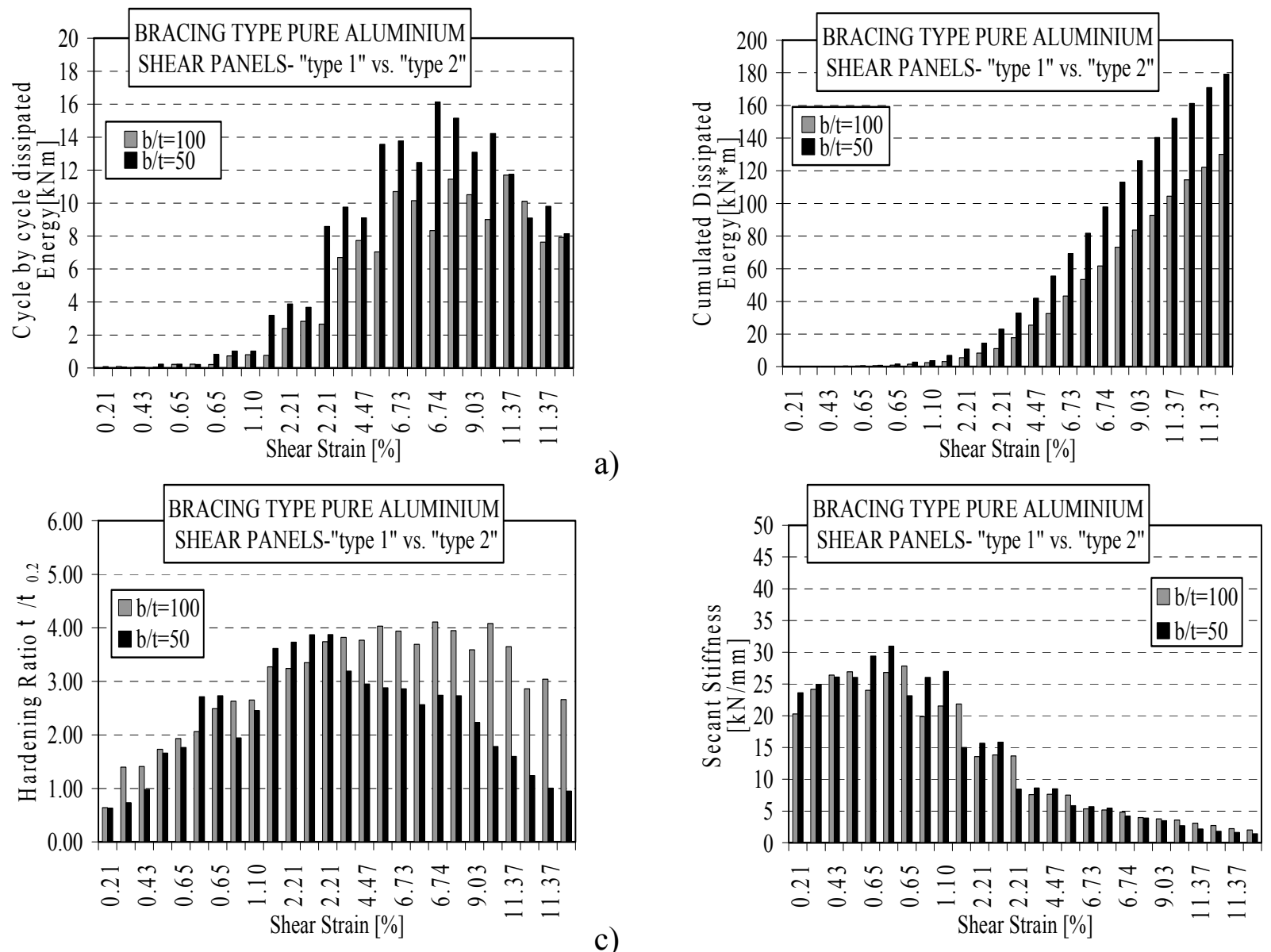

b) 


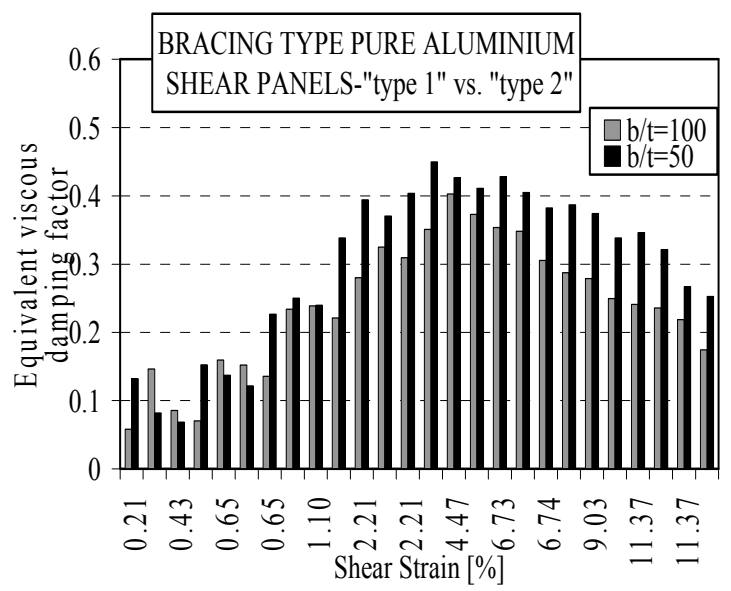

e)

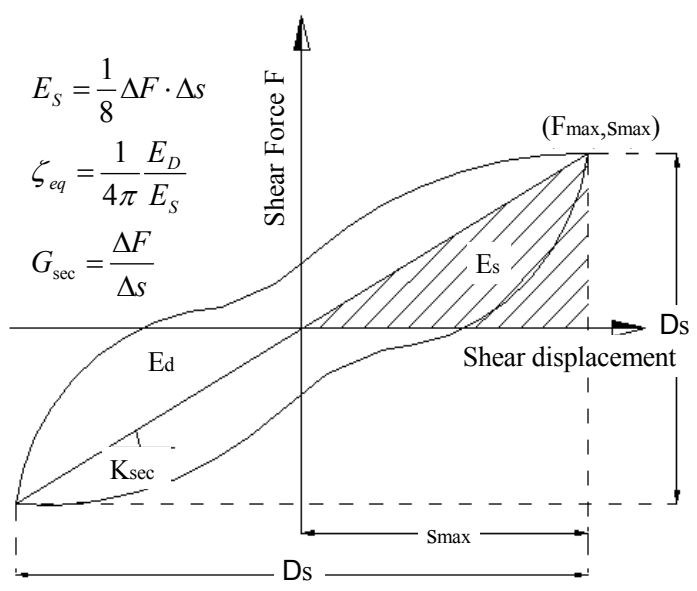

Figure 10. Comparison of Results: (a) Dissipated Energy Per Cycle; (b) Cumulated Energy;

(c) Hardening Ratio; (d) Secant Shear Stiffness; (e) Equivalent Viscous Damping Factor;

(f) Definition of Dissipated Energy $\left(\mathrm{E}_{\mathrm{s}}\right)$, Equivalent Viscous Damping $\left(\mathrm{z}_{\text {eq }}\right)$ and Secant Shear Stiffness $\left(\mathrm{G}_{\mathrm{sec}}\right)$

It is worthy noting that both systems provide a very high dissipative capability, testified by maximum equivalent viscous damping ratio values of around 0.4 which are achieved for a shear strain of $6.73 \%$ (corresponding to a diagonal displacement amplitude of $30 \mathrm{~mm}$ ). However, the panel configuration "type 1" exhibited a higher value at collapse of both the hardening ratio and the secant shear stiffness. This was mostly due to the influence provided by the perimeter connecting system. In fact, it has to be observed that panel "type 2" is characterized by a higher resistant peak, which is correlated directly to a secondary resistant mechanism in which both the transversal and longitudinal intermediate ribs operate as a sort of framework axially stressed working in parallel with the shear plate. It means that bolted connections are more stressed, anticipating their failure respect to shear panel configuration "type 1". Obviously, a better performance of the proposed devices could be obtained by applying an improvement to the connection manufacture. Anyway, it is apparent that in terms of cumulated energy and equivalent viscous damping factor, the panel "type 2" behaved better than panel "type 1", due to the beneficial effect of the stiffeners. On the other hand, this aspect is partly balanced by the higher fabrication cost of panel "type 2".

As far as the experimental evidences are concerned, three principal behavioural phases can be recognised. In the first one no buckling phenomena occurred and a pure shear resisting mechanism was developed. Analyzing the strain gauges measurements in Figure 11, which are expressed as a function of time, it is clearly evident that the first buckling phenomena arose after about 100 seconds and 200 seconds from the test starting, for panel "type 1" and panel "type 2", respectively.
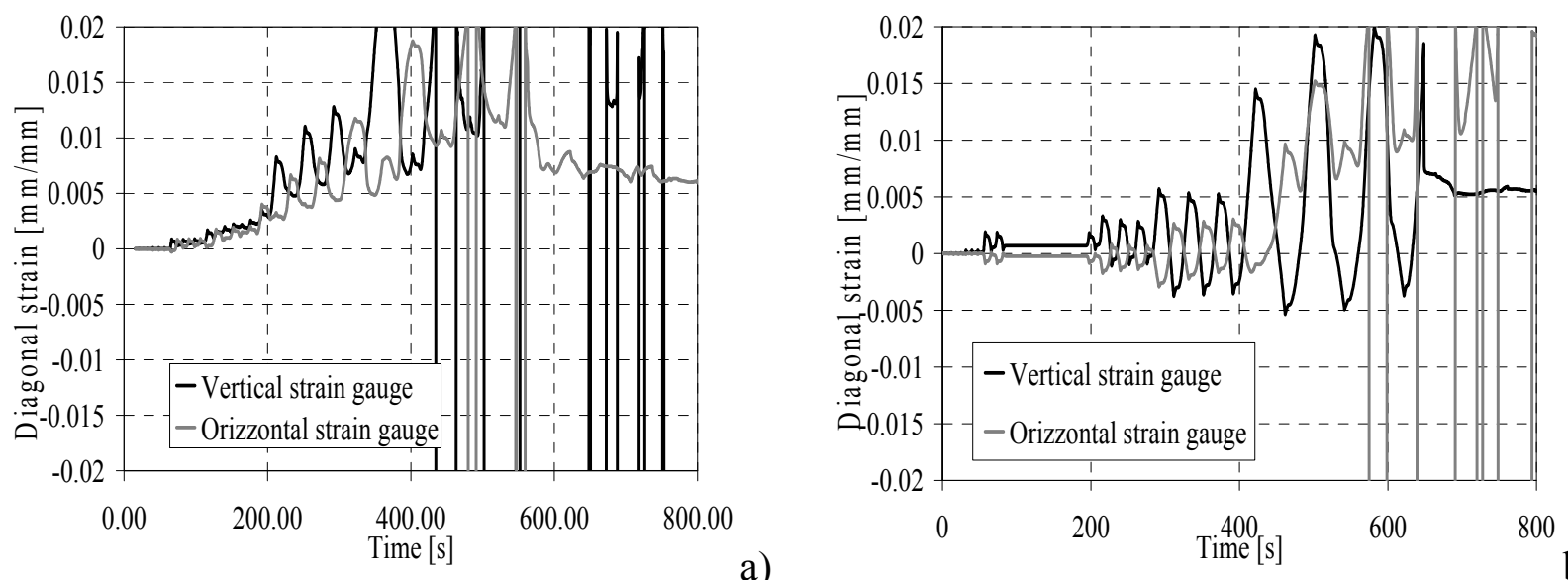

Figure 11. Strain Gauges Measurements: (a) Shear Panel "type 1"; (b) Shear Panel "type 2" 
Considering the experimental layout shown in Figure $7 \mathrm{a}$, it is possible to determine the diagonal displacements corresponding to the above mentioned testing times, they corresponding to a displacement amplitude equal to $\pm 2.00 \mathrm{~mm}$ and $\pm 5.00 \mathrm{~mm}$ for panel "type 1 " and panel "type 2", respectively. The detailed analysis of the initial phase of the test as shown in Figure 11-a and Figure 11-b gives an additional information on the yielding achievement (see Figure 12). In fact, considering a linear elastic behaviour of the base material with a Young's modulus E= $66000 \mathrm{MPa}$, it is possible to fix a strain level corresponding to the attainment of the conventional yielding stress (18 MPa) equal to:

$\varepsilon_{y}=\frac{f_{0.2}}{E}=\frac{18}{66000}=0.000272$

Such a strain value is achieved after 40-60 seconds of time testing, which correspond to a diagonal displacement of $0.5 \mathrm{~mm}$, this being the value of the elastic displacement $\left(v_{\mathrm{y}}\right)$ assumed to define the applied loading protocol discussed in Section 3.
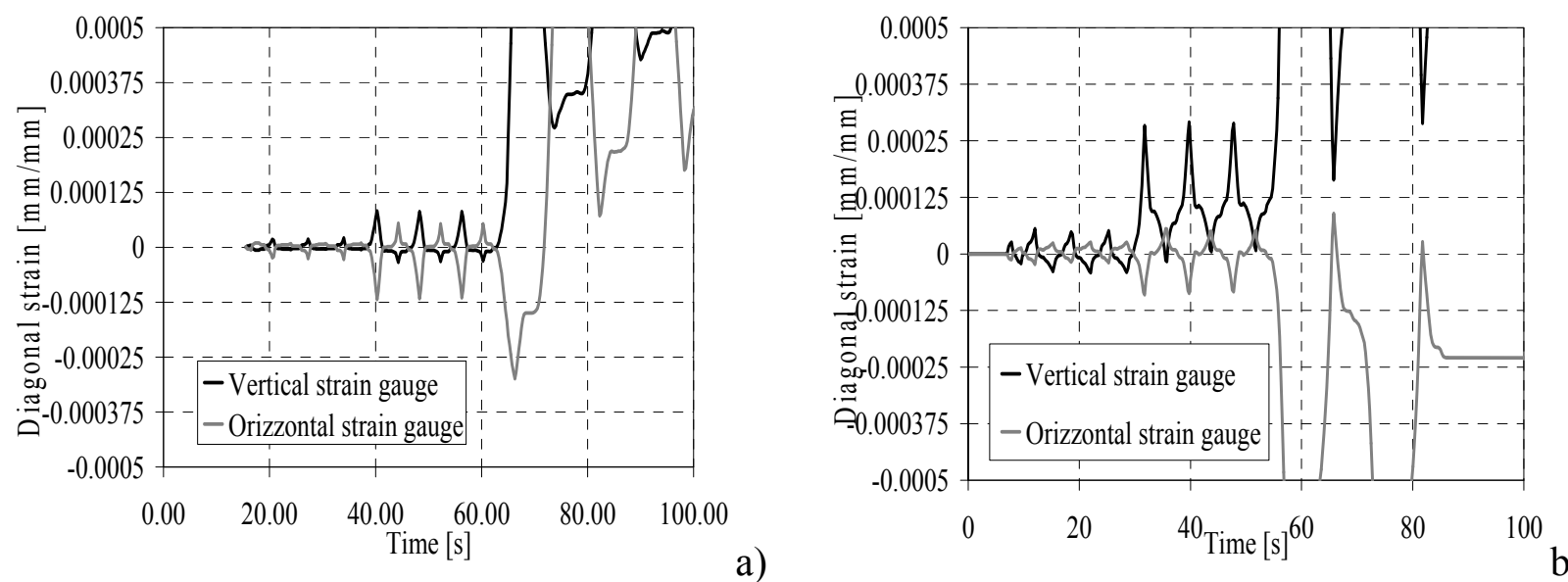

Figure 12. Elastic Strain Gauges Measurements: (a) Shear Panel "type 1"; (b) Shear Panel "type 2"
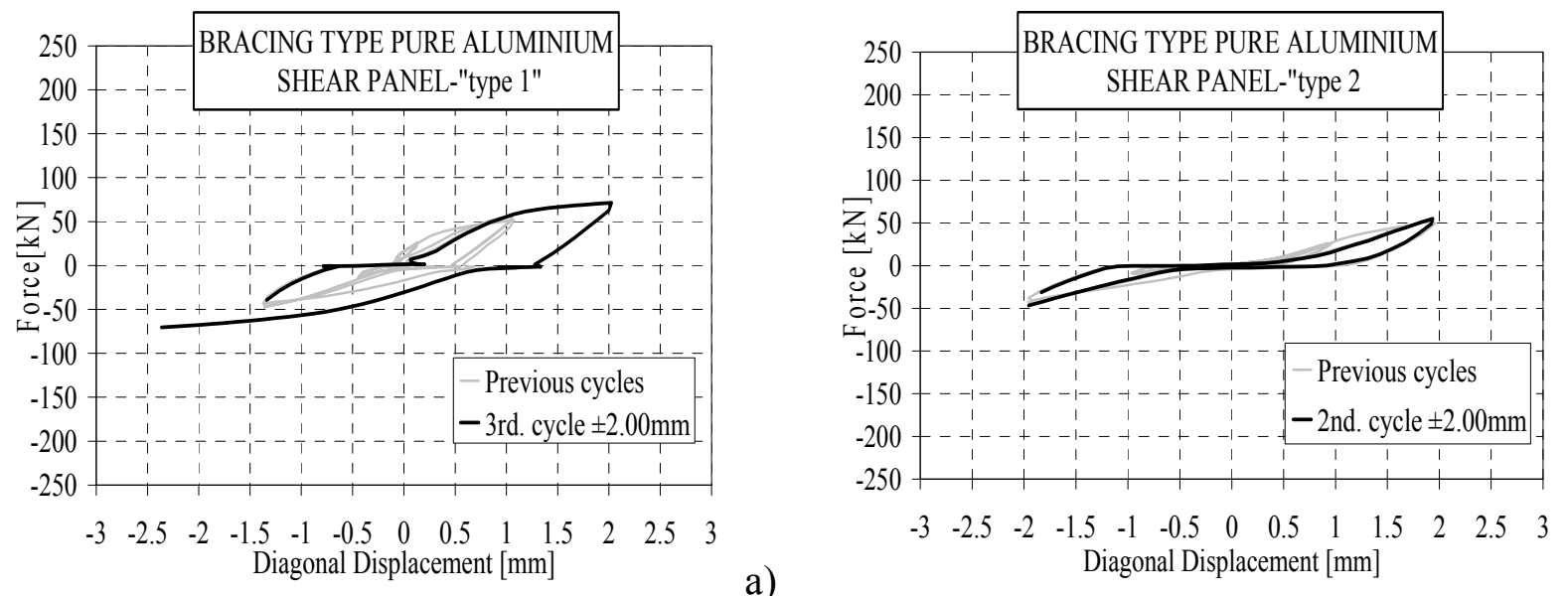

a)

b)

Figure 13. First Behavioural Phase; $\pm 2.00 \mathrm{~mm}$ Amplitude Hysteretic Cycles :

(a) Shear Panel "type 1"; (b) Shear Panel "type 2" 
In Figure 13, two characteristic hysteretic cycles of the first behavioural phase, corresponding to a diagonal displacement of $\pm 2.00 \mathrm{~mm}$, are shown for both panel "type1" and panel "type 2". These cycles allow the determination of the initial slipping of $\pm 0.70 \mathrm{~mm}$ for shear panel "type 1 " and $\pm 1.25 \mathrm{~mm}$ for shear panel "type 2", respectively.

The second behavioural phase ranges between diagonal displacement amplitudes of $\pm 5.00 \mathrm{~mm}$ and $\pm 40.00 \mathrm{~mm}$. In this case, buckling phenomena are triggered off and develop along two diagonal bands, where significant out of plane deformations are produced. Pinching effects are clearly visible in the hysteretic cycles starting from a diagonal displacement amplitude of $\pm 10.0 \mathrm{~mm}$ for panel "type 1" (Figure 14-a) and $20.0 \mathrm{~mm}$ for panel "type 2" (Figure 14-b). In Figure 14-c and Figure 14-d two characteristic hysteretic cycles registered at the end of the above second behavioural phase are shown, while in Figure 14-e and Figure 14-f buckle configurations of the panels for a displacement amplitude of $\pm 30.0 \mathrm{~mm}$ are depicted.

In the third behavioural phase, the diagonal displacement amplitude ranges between $\pm 40.0 \mathrm{~mm}$ and $\pm 50.0 \mathrm{~mm}$. The collapse of the perimetric connection system, whose bearing capacity was exceeded in the previous behavioural phase, is evidenced as well as the failure of the shear plate in the zone where the buckled bands developed in the second behavioural phase intersected each other (see Figures 8 and 9). A significant loss of resisting strength, up to 50\%, is detectable when the collapse of the whole systems is attained.
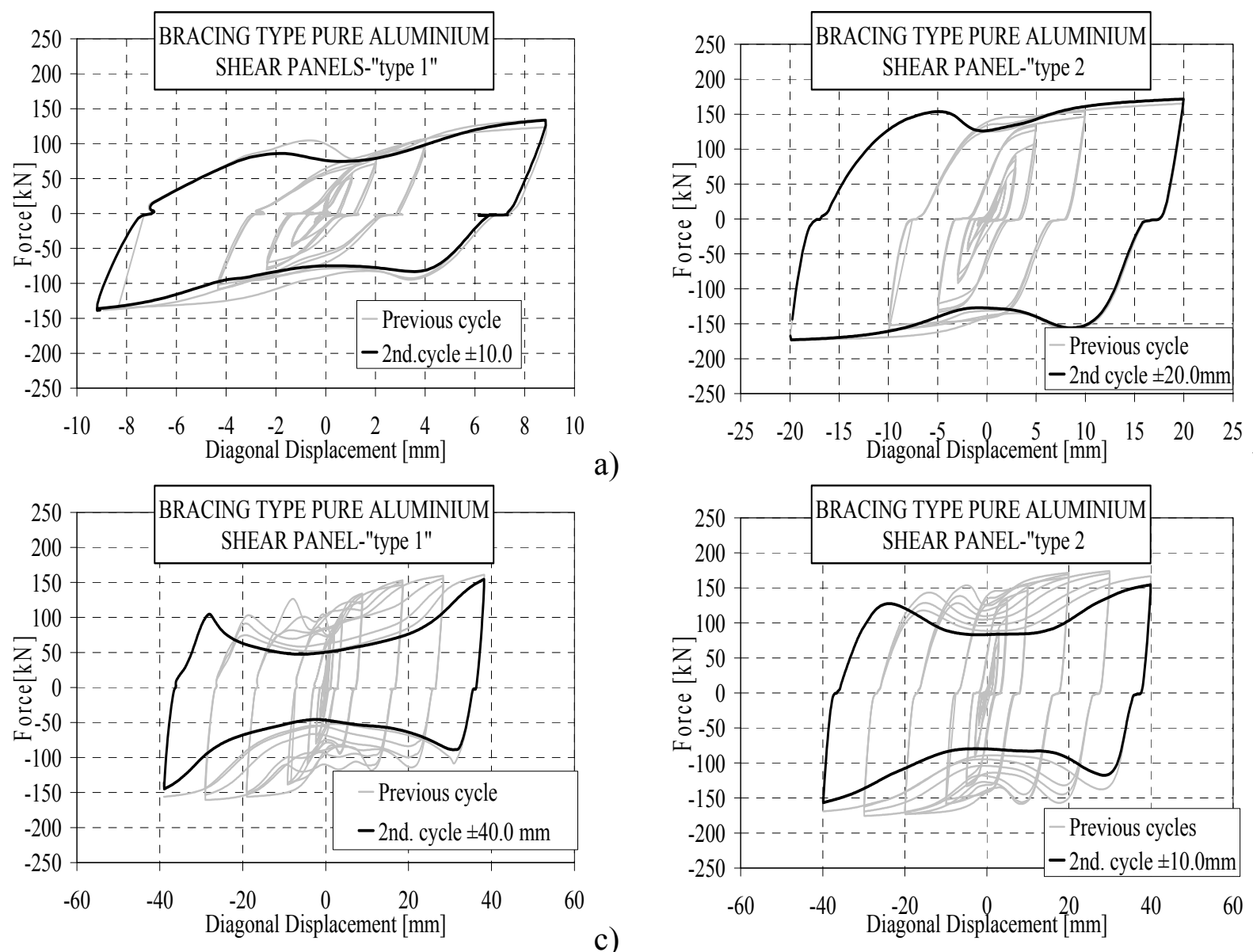

b)

c) 

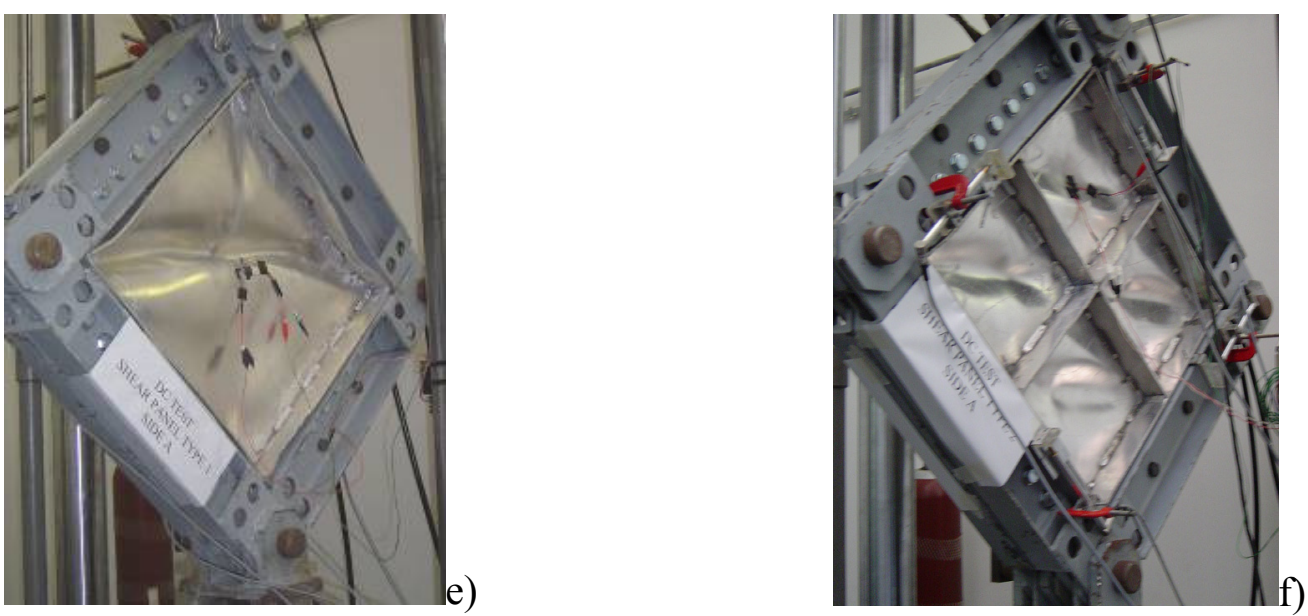

Figure 14. Second Behavioural Phase; Hysteretic Cycles: (a-c) Shear Panel "type 1"; (b-d) Shear Panel "type 2". Buckled Shapes for Displacement Amplitude of \pm 30.00 : (e) Shear Panel "type 1";

(f) Shear Panel "type 2"

\section{CONCLUSIONS}

In the current paper, experimental tests on two bracing type pure aluminium shear panels have been presented and deeply analyzed. In addition, considering the innovative features of the proposed base material, monotonic and cyclic tests on the adopted pure aluminium alloy have been also shown.

The tests were carried out in order to assess the suitability of the shear panels to be used as passive control systems in frame structures. It has been ascertained that, compared with a full bay type solution, the bracing type AW 1050A aluminium alloy shear panels provide a better dissipative behaviour, which is characterized by maximum equivalent viscous damping ratios up to 0.4 , for inter-story drift values which are of concern for common structural applications. Hence it has been emphasised that the response of both systems is characterised by three main behavioural phases (initial elastic, plastic shear buckling and collapse). Such phases have been detected qualitatively, on the basis of the exhibited resisting mechanism, but also quantitatively, by the measured diagonal displacement amplitudes corresponding to the attainment of the yielding stress and the first buckling phenomenon. In particular, the obtained experimental results have evidenced that the shear panel configuration having a geometric slenderness $a_{w} / t_{w}=50$ presents more stable and larger hysteretic cycles, while the shear panel with $a_{w} / t_{w}=100$ is characterised by a higher value of ultimate hardening ratio and shear global stiffness, as the presence of internal ribs in shear panel "type 2" determines higher stresses for the connecting system.

From this point of view further developments could be addressed to investigate more refined solutions, characterised by shear panels with different stiffener arrangements and with improved connection details. 


\section{REFERENCES}

[1] Dargush, G.F. and Soong T.T., "Behaviour of Metallic Plate Dampers in Seismic Passive Energy Dissipation Systems", Earthquake Spectra, 1995, Vol. 11, pp. 545-568.

[2] Chen, Z., Ge, H. and Usami, T., "Hysteretic Models of Stiffened Shear Panel Dampers", Journ. Struct. Engrg., ASCE, 2006, Vol. 3, pp. 478-483.

[3] Nakashima, M., "Strain-Hardening Behaviour of Shear Panels Made of Low-Yield Steel. I: Model”, Journ. Struct. Engrg., ASCE, 1995 (a), Vol. 121, No. 12, pp. 1742-1749.

[4] Nakashima, M., Akazawa, T. and Tsuji, B., "Strain-Hardening Behaviour of Shear Panels Made of Low-Yield Steel. II: Test”, J. Struct. Engrg, ASCE, 1995 (b), Vol. 121, No. 12, pp. 1750-1757.

[5] Nakagawa, S., Kihara, H., Torii, S., Nakata, Y., Matsuoka, Y., Fujisawa, K. and Fukuda, K., "Hysteretic Behaviour of Low Yield Strength Panel Shear Walls-Experimental Investigation", Proceedings of the 11th WCEE, 1996, Acapulco, Mexico.

[6] De Matteis, G., Mazzolani, F.M. and Panico, S., "Pure Aluminium Shear Panels as Passive Control System for Seismic Protection of Steel Moment Resisting Frames", Proceedings of the IV International Conference STESSA '03 (Behaviour of Steel Structures in Seismic Areas), Balkema, 2003, pp. 609-614, Naples, Italy.

[7] De Matteis, G., Formisano, A., Panico S. and Mazzolani, F.M., "Numerical and Experimental Analysis of Pure Aluminium Shear Panels with Welded Stiffeners", Computers \& Structures, 2008, Vol. 86, No. 6, pp. 545-555.

[8] De Matteis, G., Mazzolani, F.M. and Panico, S., "Pure Aluminium Shear Panels as Dissipative Devices in Moment-Resisting Steel Frames", in Earthquake Engineering and Structural Dynamics, Wiley InterScience, DOI: 10.21002/eqe, Vol. 36, pp. 841-859, $2007 \mathrm{~b}$.

[9] RILEM TC 83-CUS Testing Recommendation, "Tension Testing of Metallic Structural Material for Determining Stress-Strain Relations under Monotonic and Uniaxial Tensile Loading", Material and Structures, 1989, Vol. 23, No. 133, pp. 35-46.

[10] Katayama, T., Ito, S., Kamura, H., Ueki, T. and Okamoto, H., "Experimental Study on Hysteretic Damper with Low Yield Strength Steel Under Dynamic Loading”, Proceedings of the 12th WCEE, 2000, Auckland, New Zealand.

[11] Höglund, T., "Shear Buckling Resistance of Steel and Aluminium Plate Girders", Thin-Walled Structures, 1997, Vol. 29, No. 1-4, pp 13-30.

[12] EUROCODE 9, "Design of Aluminium Structures prEN 1999-1-1, Final Draft", 2006.

[13] ECCS-CECM, "Recommended Testing Procedure for Assessing the Behaviour of Structural Steel Elements Under Cyclic Loads", 1985. 